\title{
Cien duodenopancreatectomías cefálicas laparoscópicas. Experiencia de dos grupos de trabajo
}

\author{
Lucio Uranga, ${ }^{1,4}$ Gustavo Kohan, ${ }^{2,4}$ Luciano Bisio, ${ }^{1}$ OrnellaAgnes Ditulio, ${ }^{2}$ Jorge Omar Monestés, ${ }^{1}$ \\ Ángela Leonor Carbajal Maldonado,, ${ }^{4}$ Gabriel Raffín,, ${ }^{2}$ Fernando Duek, ${ }^{2}$ Javier Lendoire, ${ }^{2}$ \\ Oscar Inventarza ${ }^{2}$
}

\author{
${ }^{1}$ Sección Higado, Vias Biliares y Páncreas. Servicio de Cirugía. Hospital de Gastroenterología Dr. Carlos Bonorino Udaondo. \\ ${ }^{2}$ Sector de Cirugia Pancreática, Sanatorio Mitre. \\ ${ }^{3}$ Sector de Cirugía Hepatobiliopancreática y Trasplante Hepático, Sanatorio Mitre. \\ ${ }^{4}$ Sector de Cirugía Pancreática, Sanatorio Güemes. \\ Ciudad Autónoma de Buenos Aires, Argentina.
}

Acta Gastroenterol Latinoam 2020;50(2):109-117

Recibido: 16/06/2019 / Aceptado: 06/05/2020 / Publicado online: 29/06/2020

\section{Resumen}

La duodenopancreatectomía cefálica laparoscópica es un desafio para el cirujano debido a su dificultad técnica. La curva de aprendizaje es prolongada y dificultosa. Objetivo. Mostrar la experiencia conjunta de dos equipos quirúrgicos en más de cien duodenopancreatectomías cefálicas laparoscópicas. Material y método. Se analizaron todos los pacientes tratados mediante una DPCL por dos cirujanos especialistas en el periodo comprendido entre mayo de 2012 y mayo de 2018. Criterios de inclusión: pacientes con patología periampular con indicación de duodenopancreatectomía cefálica. Criterios de exclusión: pacientes con compromiso

Correspondencia: Lucio Segundo Uranga

Av. Caseros 2061, Hospital de Gastroenterología Dr. Carlos Bonorino

Udaondo, Ciudad Autónoma de Buenos Aires, Argentina

Tel.: 011-34275620

Correo electrónico: luciouranga@gmail.com vascular evidente por imágenes, con enfermedad a distancia o con inadecuado performance status. Resultados. Se realizó duodenopancreatectomía cefálica laparoscópica en 108 pacientes; 61 cirugías fueron totalmente laparoscópicas, 35 fueron cirugías hibridas, y 12 fueron convertidas a cirugía abierta. Existió un descenso significativo del tiempo operatorio a partir del caso 30. Doce cirugias $(11,3 \%)$ fueron convertidas. La fistula pancreática ocurrió en 35 de los 108 pacientes $(32,4 \%)$. Veintiséis pacientes presentaron fistula pancreática tipo $A(24,2 \%)$ y 9 (8,3\%) tuvieron fistula $B / C$. La tasa de fistula $B / C$ fue del $8,2 \%$ en anastomosis pancreática por vía laparoscópica, $8,6 \%$ en los reconstruidos por vía minilaparotómica y $8,3 \%$ en los pacientes convertidos. Requirieron alguna reintervención (Dindo-Clavien 3) 19 pacientes (17,5\%). La mortalidad fue 4,6\% $(n=5)$, dos dentro de los 30 días y tres a los 90 días. La mediana de internación total fue de 8,5 dias (rango: 4-83). Conclusión. La duodenopancreatectomía cefálica laparoscópica es un procedimiento viable y reproducible que debe aplicarse en casos seleccionados durante la curva de aprendizaje, incrementando su aplicación en la medida que aumenta el número de operaciones realizadas.

Palabras claves. Duodenopancreatectomía laparoscópica, cirugía pancreática, curva de aprendizaje. 
Laparoscopic pancreatoduodenectomy. Initial experience of two working groups

\section{Summary}

Laparoscopic pancreatoduodenectomy is a challenge for the surgeon due to its technical difficulty. The learning curve is long and difficult. Objective. To show the joint experience of two surgeons in more than 100 laparoscopic pancreatoduodenectomy. Material and methods. We analysed all patients treated by laparoscopic pancreatoduodenectomy by two specialist surgeons in the period between May 2012 and May 2018. Inclusion criteria: patients with periampullary disease with indication of pancreatoduodenectomy. Exclusion criteria: patients with evident vascular compromise by images, metastatic disease or bad performance status. Results. Laparoscopic pancreatoduodenectomy was performed in 108 patients; 61 surgeries were totally laparoscopic, 35 were hybrid surgeries, and 12 were converted to open surgery. There was a significant decrease in operative time from case number 30. Twelve surgeries (11.3\%) were converted. Pancreatic fistula occurred in 35 of 108 patients (32.4\%). Twenty-six patients presented pancreatic fistula type A (24.1\%), and 9 (8.3\%) fistula $B / C$. The $B / C$ fistula rate was $8.2 \%$ in laparoscopic pancreatic anastomosis, $8.6 \%$ in those reconstructed by mini-laparotomy and $8.3 \%$ in the converted patients. Reoperation was required (Dindo-Clavien 3) in 19 patients (17.5\%). Mortality was 4.6\% (5 patients), 2 within 30 days and 3 within 90 days. The median total hospital stay was 8.5 days (range: 4-83). Conclusion. Laparoscopic pancreatoduodenectomy is a viable and reproducible procedure that should be applied in selected cases during the learning curve, increasing its application as the number of operations carried out increases.

Key words. Laparoscopic pancreatoduodenectomy, pancreatic surgery, learning curve.

La duodenopancreatectomía cefálica laparoscópica (DPCL) es un desafío para el cirujano debido a que es técnicamente demandante tanto en la etapa resectiva como en la reconstructiva.

Su aplicabilidad y reproducibilidad son discutidas ya que dependen enteramente de la habilidad del operador. La primera DPCL fue realizada por Michel Gagner a principios de los años noventa. ${ }^{1}$ Sin embargo, luego de realizar diez casos desaconsejó su utilización por resultar un procedimiento dificultoso que insumía muchas horas y no ofrecía beneficios claros respecto de la cirugía convencional. ${ }^{2}$ El ulterior desarrollo tanto de la tecnología biomédica como de las destrezas de los cirujanos permitió que en la actualidad se lleve a cabo con éxito el abordaje mínimamente invasivo para los tumores periampulares. ${ }^{3-5}$

No hay dudas sobre el beneficio de la mínima invasividad del abordaje laparoscópico, pero la curva de aprendizaje es prolongada y dificultosa. ${ }^{3-6}$ Esta cirugía debe ser realizada exclusivamente en centros de alta complejidad donde cirujanos formados en cirugía pancreática convencional y con gran experiencia en cirugía laparoscópica de alta complejidad concentren un alto volumen de procedimientos quirúrgicos.

El objetivo de esta presentación es mostrar la experiencia conjunta de dos grupos quirúrgicos en más de cien duodenopancreatectomías cefálicas mínimamente invasivas.

\section{Material y métodos}

Se analizó para este trabajo a todos los pacientes tratados mediante una DPCL por dos equipos quirúrgicos especializados en cirugía pancreática abierta y en cirugía laparoscópica de alta complejidad, en el período comprendido entre mayo de 2012 y mayo de 2018. Los pacientes fueron operados en el ámbito público y privado. Los datos fueron recabados en forma retrospectiva de una base de datos diseñada para tal fin.

La base de datos incluyó datos del paciente (edad, sexo, comorbilidades, cirugías previas, riesgo anestesiológico), datos de la enfermedad (motivo de consulta, tiempo de evolución, patología, pérdida de peso, colestasis, drenaje biliar preoperatorio), datos de la cirugía (abordaje, requerimiento de transfusiones, tipo de resección, tipo de anastomosis, tiempo operatorio), y también datos de la evolución postoperatoria (complicaciones, estadía en unidad de terapia intensiva y sala general, tiempo de reinicio de la ingesta oral, necesidad de nutrición enteral/ parenteral, reoperaciones y resultado histopatológico).

Por tratarse de una serie de pacientes operados por patologías heterogéneas, no se analizan datos referentes a supervivencia.

\section{Pacientes}

Criterios de inclusión: pacientes con patología periampular con indicación de duodenopancreatectomía cefálica.

Criterios de exclusión:

- pacientes con compromiso vascular evidente por imágenes que requieran en la planificación preoperatoria una resección vascular mayor;

- pacientes con contraindicación de cirugía laparoscópica por causas mórbidas determinadas en la evaluación preoperatoria; 
- pacientes con enfermedad a distancia,

- pacientes con un inadecuado performance estatus.

\section{Técnica quirúrgica}

En todos los casos se utilizó intubación orotraqueal, acceso venoso central, monitoreo invasivo de presión arterial, cateterismo vesical y profilaxis antibiótica de amplio espectro.

El paciente se colocó en decúbito dorsal con las piernas cerradas utilizando seis puertos de trabajo ubicados a nivel umbilical, en ambos flancos e hipocondrios y en fosa ilíaca derecha describiendo una concavidad superior.

El cirujano se posicionó inicialmente del lado izquierdo del paciente trabajando con el puerto umbilical y el del hipocondrio izquierdo. La cámara se ubicó en el puerto colocado en el flanco izquierdo. Una vez descartada la presencia de secundarismo, se procedió a abordar el ligamento de Treitz (excepto en pacientes con sospecha de compromiso venoso mesentérico/ portal, en quienes se abordó inicialmente el espacio supramesocólico), explorando el retroperitoneo hasta visualizar la vena cava inferior (VCI) y ambas venas renales, la porción caudal del proceso uncinado y la cara dorsal de la arteria mesentérica superior (AMS) y de la vena mesentérica superior (VMS). Luego se realizó la esqueletización del mesoyeyuno. Se seccionó el ligamento gastrocólico y luego los vasos gastroepiploicos derechos. Posteriormente se realizó la linfadenectomía del grupo 8 , y se disecó la arteria hepática común, la arteria pilórica y la arteria gastroduodenal (AGD) (Figura 1).

Figura 1. Disección de la arteria hepática, arteria gastroduodenal, arteria pilórica y vena porta luego de la resección del ganglio de la estación ganglionar número ocho. AH: arteria hepática; $A G D$ : arteria gastroduodenal; $A P$ : arteria pilórica; VP: vena porta.

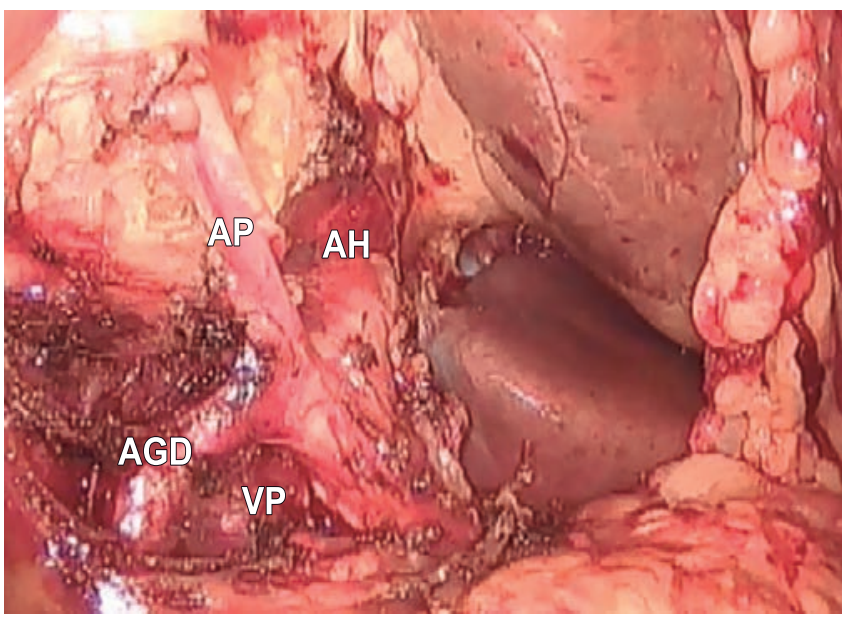

Estas últimas fueron ligadas y seccionadas, habiendo previamente corroborado el adecuado flujo en la arteria hepática bajo visión directa del latido al clampear la AGD. Esta maniobra se realizó traccionando el estómago hacia el cenit, lo cual facilitó la exposición y disección de la arteria hepática. Se seccionó el duodeno a $2-3 \mathrm{~cm}$ del píloro con una sutura mecánica lineal de $60 \mathrm{~mm}$ color azul, y se desplazó el estómago hacia la izquierda permitiendo exponer la glándula pancreática. A continuación, se identificó la vena porta por encima del borde superior del páncreas y por detrás de la arteria hepática común. Luego se disecó el borde pancreático inferior, identificando la cara anterior de la vena mesentérica superior y confeccionando el túnel retropancreático (Figura 2).

\section{Figura 2. Labrado del túnel retropancreático. VP: vena porta.}

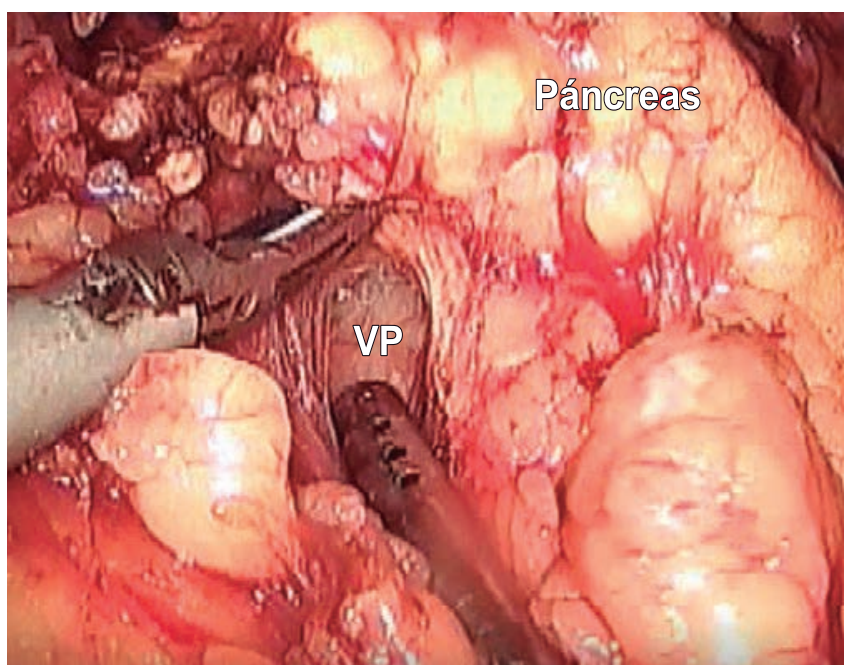

Se completó el descenso del mesocolon y ángulo hepático del colon, identificando y seccionando entre clips o ligaduras la rama gástrica del tronco venoso gastrocólico de Henle. En este momento el cirujano cambió al lado derecho del paciente, utilizando el puerto umbilical con su mano derecha y el del hipocondrio derecho para su mano izquierda. La cámara se posicionó en el puerto ubicado en el flanco derecho. Se procedió a realizar la maniobra de Votrim-Kocher. Se identificó así la primera asa yeyunal sobre la VCI y se completó el descruzamiento duodenoyeyunal. Luego se seccionó la primera asa yeyunal con una sutura mecánica lineal de $60 \mathrm{~mm}$ azul. En este momento, mediante la tracción hacia la derecha del asa yeyunal descruzada se expuso el proceso uncinado y su relación con la VMS, AMS y las adenopatías retroarteriales. La sección del proceso uncinado se realizó con dispo- 
sitivos de energía, identificando y clipando las venas tributarias de la VMS y las arterias pancreático duodenales en su nacimiento de la AMS. Se completó la sección hasta la identificación de la cara medial del colédoco. A continuación, se seccionó el páncreas a nivel de su cuello con dispositivos de energía, prestando especial atención a la identificación del conducto pancreático principal. Luego se realizó la colecistectomía, seccionando la arteria cística a nivel de su nacimiento en la arteria hepática derecha. Se completó el vaciamiento ganglionar retrocoledociano y retroportal y se seccionó la vía biliar por encima de la desembocadura del conducto cístico, previo clampeado para evitar el derrame de bilis a la cavidad. La pieza se colocó en una bolsa plástica y se cerró dentro de la cavidad, excepto que fuera necesario el estudio patológico intraoperatorio, casos en los cuales se extrajeron las piezas en ese momento.

Para la reconstrucción el cirujano permaneció del lado derecho del paciente. El asa a anastomosar se ascendió por detrás la raíz del mesenterio. La primera anastomosis solía ser la pancreático-yeyunal. En pacientes con páncreas blando y conducto de Wirsung fino, uno de los autores optó en la etapa inicial de la serie por realizar la hepáticoyeyunoanastomosis en primer término por vía laparoscópica y luego se completó la reconstrucción mediante una técnica híbrida, emplazando una incisión mediana menor a $10 \mathrm{~cm}$ a nivel epigástrico.

La técnica quirúrgica para ambos casos fue similar. Se realizó una anastomosis ducto mucosa con 5 a 8 puntos de sutura reabsorbible monofilamento, tutorizada con un catéter de silicona de pequeño calibre (Figura 3).

Figura 3. Anastomosis pancreatoyeyunal ducto mucosa tutorizada con catéter de silicona.

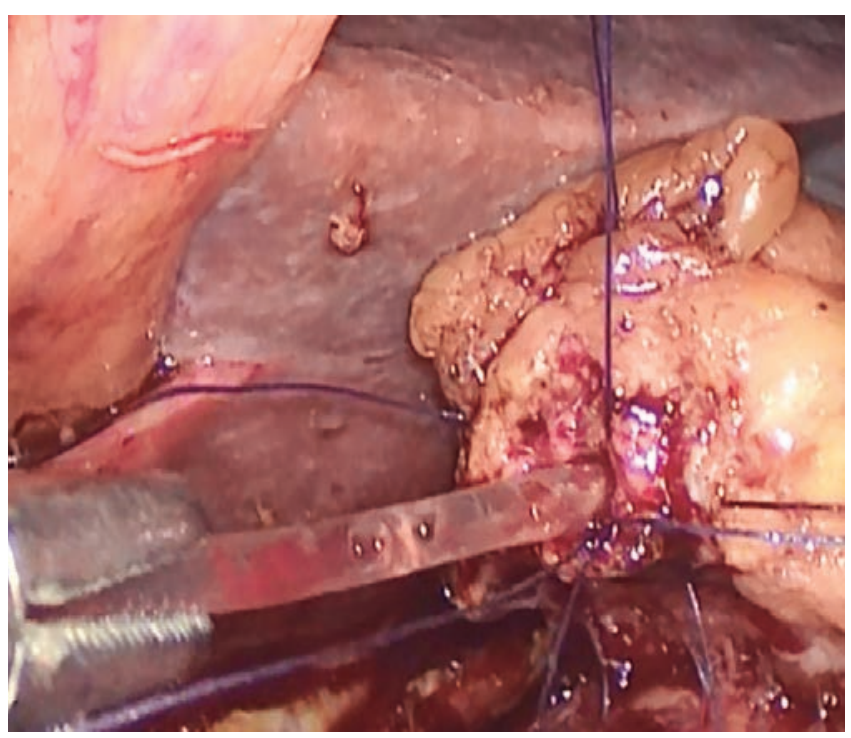

A continuación, se realizaron 2 a 4 puntos entre el páncreas (transparenquimatosos) y el yeyuno con el objeto de anclar el páncreas al yeyuno (según la técnica descripta por Blumgart).

La hepático-yeyuno anastomosis se realizó con 2 hemisurget a puntos totales de hilos 4-0 o 5-0, excepto en casos de calibres muy pequeños en los que uno de los dos cirujanos optó por realizar puntos separados. La anastomosis duodeno-yeyunal se realizó luego de extraída la pieza por una miniincisión de 4 a $5 \mathrm{~cm}$ a nivel umbilical, exteriorizando el píloro y un asa yeyunal a unos $50 \mathrm{~cm}$ de la anastomosis biliar en forma antecólica e isoperistáltica. Se realizó con puntos separados de poliglactina 3-0. Uno de los autores asoció una entero-entero anastomosis a lo Brown por la misma incisión.

No se colocó en forma sistemática yeyunostomía de alimentación. Uno de los autores dejó una sonda de alimentación nasoenteral distal a la anastomosis duodeno-yeyunal en los pacientes con una reducción de peso preoperatorio superior o igual al $10 \%$ en los últimos 6 meses. Se colocaron dos drenajes ofrecidos a las anastomosis pancreática y biliar. Se consideró conversión al requerimiento de una incisión en cualquier momento de la cirugía que no fuera planeada en forma preoperatoria.

\section{Cuidados postoperatorios}

Todos los pacientes fueron internados en la unidad de terapia intensiva. Se colocó sonda nasogástrica (SNG) en todos los casos, intentando retirarla en el primer día postoperatorio. El aporte nutricional (por vía oral o a través de la sonda nasoenteral) se inició ante la presencia de ruidos hidroaéreos. La progresión de la dieta se realizó según tolerancia.

La extracción de la sonda vesical y la deambulación se pautaron luego de la salida de la unidad cerrada de cuidados intensivos.

\section{Morbimortalidad}

Se utilizó para el diagnóstico de fístula pancreática el retardo del vaciamiento gástrico y hemorragias, la definición propuesta por el Grupo Internacional de Estudio en Cirugía Pancreática (ISGPS).

Las complicaciones fueron consignadas según la clasificación de Dindo-Clavien.

La extracción de los drenajes se realizó ante la ausencia de débito o de fístula pancreática confirmada bioquímicamente. El alta hospitalaria se otorgó a los pacientes que toleraban dieta oral, deambulaban y no presentaban signos clínicos ni bioquímicos de infección. En los pacientes con sonda nasoenteral se utilizó en forma domiciliaria hasta que cubrían requerimientos calóricos vía oral. 


\section{Análisis estadístico}

Se analizaron y compararon tres subgrupos en forma independiente: a) los pacientes tratados mediante una duodenopancreatectomía totalmente laparoscópica, b) aquellos en quienes se realizó una minilaparotomía para completar alguna maniobra quirúrgica (cirugía híbrida) y c) los pacientes convertidos a cirugía abierta.

Para el análisis comparativo fueron utilizados los test de Student para variables cuantitativas y el test de Chi o Fisher para variables cualitativas, tomándose un valor de $p$ igual o menor a 0,05 como significativo. Se utilizó para su registro y cálculos el programa Microsoft Excel, versión 16.18 .

\section{Resultados}

Ciento catorce pacientes cumplieron criterios de inclusión. Seis de ellos fueron excluidos del análisis: cuatro pacientes por presencia de metástasis hepáticas menores a un centímetro y dos por ascitis no detectada en la estadificación preoperatoria. Finalmente ciento ocho pacientes fueron incluidos. Del total, 61 cirugías fueron realizadas totalmente laparoscópicas, 35 fueron cirugías híbridas, y 12 fueron convertidas a cirugía abierta. Las variables preoperatorias registradas figuran en la Tabla 1.

Tabla 1. Variables preoperatorias.

\begin{tabular}{ll}
\hline Variable & $\mathbf{n}$ \\
\hline Sexo (F/M) n & $58 / 50$ \\
Edad mediana (rango) & $61(16-84)$ \\
DBT n (\%) & $26(24)$ \\
EPOC/TBQ n (\%) & $48(42)$ \\
HTA n (\%) & $31(28)$ \\
IMC mediana (rango) & $27(18-56)$ \\
ASA I-II n (\%) & $82(75,9)$ \\
ASA III n (\%) & $18(16,6)$ \\
Pérdida de peso n (\%) & $8(7,4)$ \\
Cirugías previas n (\%) & $45(41)$ \\
Ictericia n (\%) & $69(63)$ \\
Colangitis n (\%) & $14(13)$ \\
Ca 19,9 mediana (rango) & $343(0,8-2300)$ \\
Ecografía n (\%) & $90(83)$ \\
TC n (\%) & $96(88)$ \\
RMN n (\%) & $57(52,7)$ \\
Ecoendoscopía & $23(21,3)$ \\
Biopsia prequirúrgica n (\%) & $33(30,5)$ \\
Drenaje endoscópico n (\%) & $35(32)$ \\
Drenaje percutáneo n (\%) & $17(15,7)$ \\
\hline
\end{tabular}

Las variables operatorias se grafican en la Tabla 2. El tiempo quirúrgico fue en promedio de 429 minutos, con una mediana de 420 (rango: 330-720).

Tabla 2. Variables operatorias.

\begin{tabular}{llll}
\hline & $\begin{array}{l}\text { Laparoscópica } \\
(\mathrm{n}=61)\end{array}$ & $\begin{array}{l}\text { Híbrida/ } \\
\text { convertida } \\
(\mathrm{n}=47)\end{array}$ & $\boldsymbol{p}$ \\
\hline Consistencia dura & 31 & 13 & 0,018 \\
Consistencia & 30 & 34 & NS \\
blanda/intermedia & 37 & 27 & NS \\
Vía biliar dilatada & 60 & 35 & NS \\
Preservación píloro & 57 & 47 & NS \\
PYA ducto-mucosa & 4 & 0 & NS \\
PYA intususceptada & 51 & 43 & NS \\
Tutor Wirsung & 5 & 5 & NS \\
Drenaje Biliar & 490 & 320 & $<0,001$ \\
Tiempo qx (mediana) & & & NS \\
UGR (mediana) & 5 & & \\
\hline
\end{tabular}

NS: no significativo.

La curva de aprendizaje se realizó en forma diferente entre los equipos quirúrgicos. Uno de los grupos realizó las primeras 13 cirugías con técnica híbrida. Una vez que se logró reducir el tiempo quirúrgico, empezaron a realizarse los abordajes totalmente laparoscópicos en los pacientes con un páncreas de consistencia dura/intermedia, y posteriormente se operó por la vía puramente laparoscópica a todos los pacientes seleccionados.

El otro grupo de trabajo inició los primeros 10 casos de su serie con pacientes portadores de lesiones pequeñas alejadas del eje vascular (ampulomas y tumores de vía biliar distal), y a partir del caso 11 se ofreció el abordaje totalmente laparoscópico a pacientes con tumores de mayor tamaño.

Existió un descenso significativo del tiempo operatorio a partir del caso 30 en ambos grupos ( 480 vs. 390 minutos; $p=0,005$ ) (Figura 4). El requerimiento de hemoderivados de toda la serie presentó un promedio de 0,45 UGR (0-5). 
Figura 4. Evolución del tiempo operatorio.

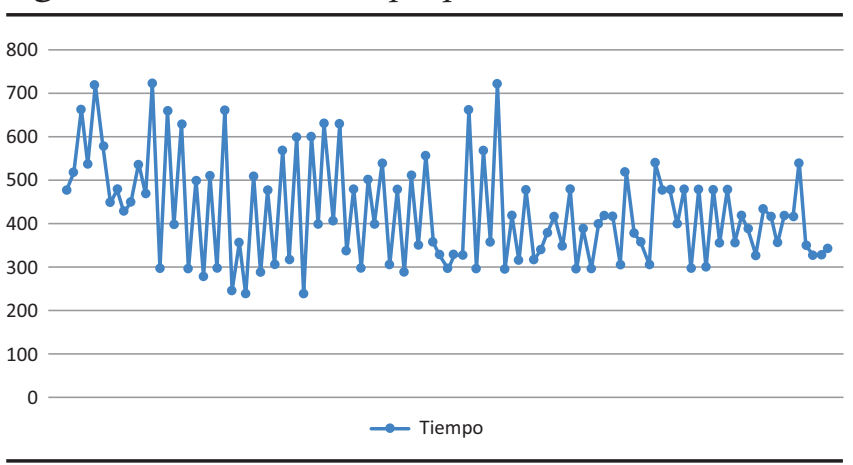

El desarrollo de una fístula pancreática se presentó en 35 de los 108 pacientes (32,4\%). Veintiséis pacientes presentaron fístula pancreática tipo A, y $9(8,3 \%)$ fueron clínicamente relevantes (grado $\mathrm{B}$ o $\mathrm{C}$ ).

La tasa de fístula $\mathrm{B}$ o $\mathrm{C}$ fue de $8,2 \%$ (5/61 pacientes) en los pacientes sometidos a una anastomosis pancreática por vía laparoscópica, 8,57 \% (3/35 pacientes) en los reconstruidos por vía minilaparotómica y $8,3 \%(1 / 12)$ en los pacientes convertidos. En 4 pacientes del grupo laparoscópico no logró identificarse el conducto pancreático, por lo que se realizó una anastomosis telescopada no tutorizada, y 2 de estos pacientes desarrollaron una fístula gra- do B. El resto de las anastomosis fueron ducto mucosas. La tasa de fístula fue significativamente menor $(p=0,04)$ en los páncreas duros comparados con los de consistencia blanda o intermedia ( 44 vs. 64 pacientes), independientemente de la vía de abordaje. No se presentaron fístulas clínicamente relevantes en pacientes con páncreas duros (Tabla 3).

Tabla 3. Prevalencia de fístula según la consistencia del páncreas.

\begin{tabular}{lll}
\hline Consistencia & Duro & Blando/intermedio \\
$\mathrm{n}$ & 44 & 64 \\
Fístula A n (\%) & $6(13,6)$ & $20(31,2)$ \\
Fístula B/C n (\%) & $0(0,0)$ & $9(14,0)$ \\
Total de fístulas n (\%) & $6(13,6)$ & $29(45,3)$ \\
\hline
\end{tabular}

La tasa de retardo del vaciamiento gástrico fue del $12,9 \%$ y de hemorragia postoperatoria del 4,6 \%. En la Tabla 4 se detallan las variables postoperatorias evaluadas.

Requirieron reintervención quirúrgica (Dindo-Clavien 3) un total de 19 pacientes (17,5\%) (Tabla 5).

Tabla 4. Variables postoperatorias.

\begin{tabular}{|c|c|c|c|c|c|}
\hline Variable & $\mathrm{n}$ & Laparoscópica & Híbrida & Convertida & $\boldsymbol{p}$ (lap vs. híbrido) \\
\hline N & 108 & 61 & 35 & 12 & \\
\hline Pancr. duro n \% & $44(40,7)$ & $31(50,8)$ & $8(22,8)$ & $5(41,6)$ & 0,0479 \\
\hline Fístula pancr. tipo A n \% & $26(24)$ & $15(24,5)$ & $8(22,8)$ & $3(25)$ & NS \\
\hline Fístula (B/C) n \% & $9(8,3)$ & $5(8,19)$ & $3(8,57)$ & $1(8,3)$ & NS \\
\hline $\operatorname{RVG}(\mathrm{B} / \mathrm{C}) \mathrm{n} \%$ & $14(12,9)$ & $9(14,7)$ & $4(11,42)$ & $1(8,3)$ & NS \\
\hline Bilirragia n \% & $5(4,6)$ & $4(6,5 \%)$ & $1(2,8)$ & 0 & NS \\
\hline Dehiscencia DYA & $2(1,8)$ & $2(3,3)$ & 0 & 0 & NS \\
\hline Hemorragia n \% & $5(4,6)$ & $2(3,27)$ & $2(5,7)$ & $1(8,3)$ & NS \\
\hline (Clavien 1-2) n \% & $26(23,8)$ & $15(24,5)$ & $7(20)$ & $4(33,3)$ & NS \\
\hline Reoperación n \% & $19(17,4)$ & $10(16,3)$ & $7(20)$ & $2(16,6)$ & NS \\
\hline Reinternación n \% & $11(10,6)$ & $6(9,83)$ & $4(11,4)$ & $1(8,3)$ & NS \\
\hline Estadía mediana (rango) & $8,5(4-42)$ & $6(4-42)$ & $10(5-83)$ & $10(7-30)$ & NS \\
\hline Mortalidad 30 días n \% & $2(1,8)$ & $1(1,6)$ & $1(2,8)$ & 0 & NS \\
\hline Mortalidad 90 días n \% & $3(2,7)$ & $3(4,9)$ & 0 & 0 & NS \\
\hline Mortalidad total n \% & $5(4,6)$ & $4(6,5)$ & $1(2,8)$ & 0 & NS \\
\hline
\end{tabular}

NS: no significativo. 
Tabla 5. Reintervenciones quirúrgicas.

\begin{tabular}{lc}
\hline Reoperación & $\mathrm{n}$ \\
Fístula pancreática B & 3 \\
Fístula pancreática C & 6 \\
Sangrado & 4 \\
Fiebre/dolor abdominal & 1 \\
Vólvulo de intestino delgado & 1 \\
Isquemia segmentaria de intestino delgado & 1 \\
Dehiscencia duodenoyeyuno & 2 \\
Bilirragia & 1 \\
\hline
\end{tabular}

Diez pacientes $(15,3 \%)$ pertenecían al grupo laparoscópico, 6 al grupo híbrido $(19,3 \%)$ y 3 correspondían al grupo de pacientes convertidos (25\%). La mediana de internación total fue de 8,5 días (rango: 4-83).

Doce cirugías $(11,3 \%)$ fueron convertidas y las causas fueron:

1. Hemorragia de ramas venosas afluentes de la vena porta: 4 pacientes.

2. Compromiso vascular venoso: 6 pacientes.

3. Dificultad anatómica: 2 pacientes.

\section{Mortalidad}

Fallecieron 5 pacientes $(4,6 \%)$. Dos pacientes dentro de los primeros 30 días postoperatorios:

1. El primer paciente fue un hombre de 60 años operado por un adenocarcinoma de páncreas por vía totalmente laparoscópica. Fue externado al quinto día postoperatorio y presentó al día 12 shock hipovolémico en el domicilio. Se reintervino quirúrgicamente y se observó sangrado de la arteria gastroduodenal. El paciente evolucionó con una falla multiorgánica y falleció al día 14 del postoperatorio.

2. El segundo fue un paciente de 52 años tratado mediante una duodenopancreatectomía cefálica por un tumor ampular con técnica híbrida. Desarrolló una fístula pancreática grado C, por lo que fue reintervenido y falleció al décimo día postoperatorio por falla multiorgánica.

Tres pacientes fallecieron dentro de los 90 días postoperatorios:

1. La primera fue una mujer de 62 años tratada con
DPCL por ampuloma. Evolucionó con una fistula biliar y retardo del vaciamiento gástrico por lo que se reintervino quirúrgicamente al trigésimo segundo día postoperatorio. Evolucionó con accidente cerebro vascular hemorrágico, y falleció al día 35 postoperatorio.

2. El segundo paciente fue un hombre de 81 años con cardiopatía chagásica, operado por un carcinoma ampular por vía totalmente laparoscópica. $\mathrm{Al} 2^{\text {do }}$ día postoperatorio presentó fístula pancreática y evolucionó con insuficiencia cardíaca congestiva y edema agudo de pulmón. Fue reoperado por la fístula pancreática. Falleció el día 41 postoperatorio por infarto agudo de miocardio.

3. La tercera paciente fue una mujer de 72 años con un tumor neuroendocrino de cabeza de páncreas, que fue operada por vía totalmente laparoscópica. Evolucionó con una fístula grado $\mathrm{B}$ que requierió un drenaje percutáneo y se reoperó por fístula pancreática no dirigida. Falleció el día 35 del postoperaotorio por falla multiorgánica.

\section{Histopatología}

De los 108 pacientes, 87 (80,5\%) fueron operados por patología neoplásica. El 58,6\% (51/87) eran adenocarcinomas ductales de páncreas (Tabla 6). El tamaño tumoral promedio fue de $29,7 \mathrm{~mm}$, correspondiendo a un pT1 en el 13,3\%, pT2 en el 22,2\%, pT3 en el $60 \%$ y pT4 en el 4,4\%. El promedio de ganglios resecados fue de 18,8 y el $68,8 \%$ de los pacientes presentaban metástasis ganglionares.

Tabla 6. Diagnósticos histopatológicos.

\section{Diagnóstico}

n

Adenocarcinoma ductal

Carcinoma ampular

Colangiocarcinoma distal

IPMN

TNE

Adenocarcinoma duodenal

Pancreatitis autoinmune tipo 2

Quiste de colédoco 


\section{Discusión}

Los procedimientos miniinvasivos tienen ventajas en lo que respecta a la menor cantidad de días de internación, menor dolor postoperatorio y más rápida reinserción a las actividades habituales; sin embargo, no todos los trabajos publicados sobre DPCL reflejan las ventajas mencionadas de la miniinvasividad. La DPCL es un procedimiento cuestionado debido a su dificultad técnica ${ }^{17} \mathrm{y}$ probablemente las ventajas de la miniinvasividad puedan hacerse evidentes una vez superada la curva de aprendizaje, la cual es prolongada y dificultosa.

El trabajo de Adam, que compara la duodenopancreatectomía cefálica miniinvasiva y la realizada por vía convencional, refleja que por vía laparoscópica los pacientes comienzan a alimentarse en forma precoz y tienen una menor estadía hospitalaria. En lo que respecta a los resultados oncológicos (número de ganglios resecados, índice de R1/R0) no hubo ninguna diferencia entre ambas vías de abordaje. Sin embargo, ese estudio muestra una mayor mortalidad a los 90 días en los pacientes operados por vía laparoscópica. Eso se explica porque es un trabajo retrospectivo multicéntrico, en el cual el 92\% de las DPCL se realizaron en centros de bajo volumen (menos de 10 casos en 2 años). ${ }^{3}$ No hay dudas de que esta cirugía, técnicamente demandante, debe ser realizada en centros de alta complejidad por cirujanos con experiencia tanto en cirugía de páncreas convencional como en cirugía laparoscópica de alta complejidad. Ya fue demostrado que la morbimortalidad de la DPC se reduce cuando se realiza en centros con más de 16 DPC anuales. ${ }^{3,14,15}$ Lo mismo se aplica al abordaje laparoscópico de la DPC. Ambos grupos quirúrgicos de este trabajo realizan al menos 30 duodenopancreatectomías anuales en centros de alta complejidad y eso se refleja en los índices de mortalidad tardía aquí presentados. En lo que respecta a los resultados oncológicos, no pueden compararse los índices R1/ R0 de esta serie con los estándares internacionales dado que la patología que se incluye en este trabajo abarca patologías maligna, premaligna y benigna.

Existen tres trabajos prospectivos y randomizados que comparan DPCL y DPC abierta, con resultados controvertidos. El trabajo PADULAP ${ }^{4}$ reporta mejores resultados para la DPCL en lo que respecta a días de internación, comunicando una prevalencia de complicaciones y resultados oncológicos similares. Poves sostiene que el requerimiento fundamental para llevar a cabo una DPCL es que el cirujano interviniente tenga una amplia experiencia en cirugía laparoscópica de alta complejidad y esté muy entrenado en sutura intracorpórea. Resultados similares señala el trabajo de Palanivelu, ${ }^{5}$ también mostrando una estadía hospitalaria significativamente menor para la
DPCL. Sin embargo, el estudio LEOPARD $2^{12}$ realizado por el grupo holandés muestra resultados en detrimento de la DPCL contrariamente a los dos trabajos antes mencionados. Este estudio debió ser suspendido por la elevada mortalidad de la rama de la cirugía laparoscópica. Cinco muertes en 50 pacientes en el grupo miniinvasivo y una muerte en 49 pacientes del grupo abierto. Las causas de la mortalidad fueron cuatro complicaciones hemorrágicas (dos lesiones intraoperatorias de la arteria mesentérica superior y dos sangrados postoperatorios), y una fístula pancreática tipo C. Probablemente los sangrados intraoperatorios por lesiones vasculares mayores puedan explicarse por un problema técnico asociado a la experiencia del cirujano en DPCL. Los cuatro cirujanos que participaron en el estudio habían realizado, al menos, 20 DPCL. No está determinado el número de casos para completar la curva de aprendizaje, pero, probablemente, si el estudio se diseña con cirujanos con más de 100 DPCL los errores técnicos no se observen. Según Tseng, la curva de aprendizaje de la DPC convencional es de 60 casos. ${ }^{15}$ En la serie de este trabajo no hubo lesiones vasculares mayores intraoperatorias, como ocurrió en el estudio LEOPARD, y la morbimortalidad reportada permitió continuar realizando la duodenopancreatectomía cefálica por vía laparoscópica. Ambos grupos quirúrgicos ya tenían amplia formación en cirugía pancreática convencional y también en cirugía laparoscópica de alta complejidad, lo cual permitió realizar las cirugías con la seguridad necesaria.

La experiencia de este trabajo muestra que es factible desarrollar la DPCL con una morbimortalidad aceptable. Es difícil determinar cómo debe realizarse la curva de aprendizaje en la DPCL. No hay duda de que la práctica en modelos inanimados o en simuladores tiene una gran utilidad en el entrenamiento del cirujano. Una condición fundamental es que el operador tenga una gran experiencia en sutura intracorpórea al momento de decidir la realización de la etapa reconstructiva en la DPCL. Este entrenamiento se logra habiendo realizado derivaciones biliodigestivas laparoscópicas, gastroenteroanastomosis laparoscópicas y anastomosis cistodigestivas por vía miniinvasiva. La única diferencia en la forma de llevar a cabo la curva de aprendizaje de los dos equipos quirúrgicos fue que un grupo decidió realizar la cirugía en etapas y el otro grupo solamente seleccionando tumores de pequeño tamaño. La cirugía en etapas o cirugía híbrida consiste en realizar la resección por laparoscopía y la reconstrucción por minilaparotomía. La ventaja de la cirugía híbrida es que logra evitar tiempos prolongados durante la curva de aprendizaje. Probablemente ambas formas de aprendizaje, tanto la cirugía híbrida como la 
selección estricta de pacientes, sean válidas para comenzar a desarrollar la DPCL.

La complicación más temida de la DPCL es la fístula pancreática. En este estudio no hubo diferencia significativa en la incidencia de fístula pancreática entre las anastomosis intracorpóreas y las anastomosis por minilaparotomía. El resto de las complicaciones descriptas se encontró dentro de la incidencia de complicaciones publicadas en la literatura. Los tiempos quirúrgicos comenzaron a reducirse luego de la resección número 30 en ambos grupos, logrando en las últimas cirugías tener tiempos que superaron a los de la cirugía convencional en dos horas.

El problema de la duodenopancreatectomía cefálica es que todavía hoy es un procedimiento dependiente de la capacidad del cirujano. Kendrick ${ }^{13}$ presentó en su serie a pacientes con resección laparoscópica de arteria hepática y resección total de vena porta con interposición venosa, demostrando que la laparoscopía es factible de realizarse en cualquier paciente con buenos resultados. ${ }^{6}$ Sin embargo, la experiencia de resección vascular de Kendrick no es extrapolable ni reproducible incluso para la mayoría de los cirujanos especialistas en laparoscopía pancreática.

El método sin dudas tiene ventajas, pero es importante que cada grupo quirúrgico avance por laparoscopía en la medida de sus posibilidades, dado que este procedimiento mal utilizado puede resultar en perjuicio para el paciente. La conversión precoz no es un fracaso, todo lo contrario. La subestimación de la dificultad del método se refleja en una mayor mortalidad, en especial durante la curva de aprendizaje.

En conclusión, la presente experiencia demuestra que la DPCL es un procedimiento viable y reproducible que debe aplicarse en casos seleccionados durante la curva de aprendizaje, incrementando su indicación en la medida que aumenta el número de operaciones realizadas. Debe realizarse en centros de alto volumen en cirugía pancreática por cirujanos formados en cirugía pancreática convencional y cirugía laparoscópica de alta complejidad. No es un procedimiento que pueda aún tomarse como un estándar para toda la patología pancreática, sino que debe ser una herramienta más para el cirujano pancreático actual.

\section{Referencias}

1. Gagner M, Pomp A. Laparoscopic pylorus-preserving pancreato duodenectomy. Surg Endosc 1994; 8: 408-410.

2. Gagner M, Pomp A. Laparoscopic pancreatic resection: Is it worthwhile? J Gastrointest Surg 1997; 1: 20-25; discussion 25-26.

3. Kimura Y, Hirata K, Mukaiya M, Mizuguchi T, Koito K, Katsuramaki $\mathrm{T}$. Hand-assisted laparoscopic pylorus-preserving pancreaticoduodenectomy for pancreas head disease. Am J Surg 2005; 189: 734-737.
4. Ammori BJ. Laparoscopic hand-assisted pancreatico-duodenectomy: initial UK experience. Surg Endosc 2004; 18: 717-718.

5. Kuroki T, Kitasato A, Adachi T, Tanaka T, Hirabaru M, Matsushima H, Soyama A, Hidaka M, Takatsuki M, Eguchi S. Learning Curve for Laparoscopic Pancreaticoduodenectomy: A Single Surgeon's Experience with Consecutive Patients. Hepatogastroenterology 2014; 61: 838-841.

6. Speicher PJ, Nussbaum DP, White RR, Zani S, Mosca PJ, Blazer DG $3^{\text {rd }}$, Clari BM, Pappas TN, Tyler DS, Perez A. Defining the learning curve for laparoscopic pancreaticoduodenectomy. Ann Surg Oncol 2014; 21: 4014-4019.

7. Wang M, Meng L, Cai Y, Li Y, Wang X, Zhang Z, Peng B. Learning Curve for Laparoscopic Pancreaticoduodenectomy: a CUSUM Analysis. J Gastrointest Surg 2016; 20: 924-935.

8. Kim SC, Song KB, Jung YS, Kim YH, Park DH, Lee SS, Seo DW, Lee SK, Kim MH, Park KM, Lee YJ. Short-term clinical outcomes for 100 consecutive cases of laparoscopic pylorus preserving pancreatoduodenectomy: improvement with surgical experience. Surg Endosc 2013; 27: 95-103.

9. Adam MA, Choudhury K, Dinan MA, Reed SD, Scheri RP, Blazer DG $3^{\text {rd }}$, Roman SA, Sosa JA. Minimally invasive versus open pancreaticoduodenectomy for cancer: practice patterns and short-term outcomes among 7061 patients. Ann Surg 2015; 262 : 372-377.

10. Poves I, Burdi F, Morato O, Iglesias M, Radosevic A, Ilzarbe L, Visa L, Grande L. Comparison of Perioperative Outcomes Between Laparoscopic and Open Approach for Pancreatoduodenectomy, The PADULAP Randomized Controlled Trial. Ann Surg 2018; 268: 731-739.

11. Palanivelu C, Senthilnathan P, Sabnis SC, Babu NS, Srivatsan Gurumurthy S, Anand Vijai N, Nalankilli VP, Praveen Raj P, Parthasarathy R, Rajapandian S. Randomized clinical trial oflaparoscopic versus open pancreatoduodenectomy for periampullary tumors. Br J Surg 2017; 104: 1443-1450.

12. Rooij T, van Hilst J, Bosscha K, Dijkgraaf MG, Gerhards MF, Groot Koerkamp B, Hagendoorn J, de Hingh IH, Karsten TM, Lips DJ, Luyer MD, Molenaar IQ, van Santvoort HC, Tran TCK, Busch OR, Festen S, Besselink MG; Dutch Pancreatic Cancer Group. Minimally invasive versus open pancreatoduodenectomy (LEOPARD-2): study protocol for a randomized controlled trial. Trials 2018; 19: 1 .

13. Kendrick ML, Sclabas GM. Major venous resection during total laparoscopic pancreaticoduodenectomy. HPB (Oxford) 2011; 13: 454-458.

14. Ziegler KM, Nakeeb A, Pitt HA, Schmidt CM, Bishop SN, Moreno J, Matos JM, Zyromski NJ, House MG, Madura JA, Howard TJ, Lillemoe KD. Pancreatic surgery: evolution at a high-volume center. Surgery 2010; 148: 702-709.

15. Joseph B, Morton JM, Hernandez-Boussard T, Rubinfeld I, Faraj C, Velanovich V. Relationship between hospital volume, system clinical resources, and mortality in pancreatic resection. J Am Coll Surg 2009; 208: 520-527.

16. Tseng JF, Pisters PW, Lee JE. The learning curve in pancreatic surgery. Surgery 2007; 141: 694-701.

17. Mazza O, Sánchez Claria R, Bersano F, Yazde L, de Santibañes E, Pekolj J. Duodenopancreatectomía totalmente laparoscópica. Consideraciones técnicas y aplicabilidad inicial en un centro de alto volumen en patología pancreática. Rev Argent Ciruj 2012; 102: 62-68. 